\title{
Strong Stabilization of MIMO Systems with Restricted Zeros in the Unstable Region
}

\author{
A. N. Gündeş and H. Özbay
}

\begin{abstract}
The strong stabilization problem (i.e., stabilization by a stable feedback controller) is considered for a class of finite dimensional linear, time-invariant, multi-input multioutput plants. It is assumed that the plant satisfies the parity interlacing property, which is a necessary condition for the existence of strongly stabilizing controllers. Furthermore, the plant class under consideration has no restrictions on the poles, on the zeros in the open left-half complex plane, on the zeros at the origin or at infinity; but only one finite positive real zero is allowed. A systematic strongly stabilizing controller design procedure is proposed that applies to any plant in the class, whereas alternative approaches may work for larger class of plants but only under certain sufficient conditions. The freedom available in the design parameters may be used for additional performance objectives although the only goal here is strong stabilization. In the special case of single-input single-output plants in the class considered, the proposed stable controllers have order one less than the order of the plant.
\end{abstract}

\section{INTRODUCTION}

In this paper we discuss the strong stabilization problem for a class of linear time-invariant (LTI), multi-input multioutput (MIMO) plants that have restrictions on their zeros in the region of instability. Strong stabilization refers to output feedback stabilization of a given plant by a stable controller. Interest in the strong stabilization is due to important practical considerations as well as due to the equivalence of simultaneous stabilization of two plants to the strong stabilization of one related system [14].

Although stable stabilizing controller design is important, not all plants are strongly stabilizable. It is well known that a given plant is strongly stabilizable if and only if it satisfies the parity interlacing property (PIP); a plant is said to satisfy the PIP if the number of poles (counted according to their McMillan degrees) between any pair of blocking-zeros on the extended positive real-axis is even [15], [14].

In the case of single-input multi-output plants, and singleinput single-output (SISO) plants as a special case, several procedures are available for obtaining strongly stabilizing controllers involving interpolation constraints to construct a unit in stable rational functions and usually resulting in very high order controllers (e.g. [15], [14], [5]). A parameterization of all strongly stabilizing controllers can be obtained

This work was supported in part by TUBITAK under grant no. EEEAG$105 \mathrm{E} 156$.

A. N. Gündeş is with the Department of Electrical and Computer Engineering, University of California, Davis, CA 95616 angundes Qucdavis.edu

H. Özbay is with the Department of Electrical and Electronics Engineering, Bilkent University, Ankara, 06800 Turkey hitayabilkent.edu.tr for SISO plants using interpolation with infinite dimensional transfer functions [14]. Although extensions of these interpolation techniques to MIMO plants are not available, strong stabilization of MIMO plants has been studied extensively in the literature, some using numerical approaches and some under $H_{\infty}$ or $H_{2}$ performance criteria (e.g., [2], [3], [4], [8], [9], [12], [16], [17] and references therein). Analytical synthesis methods to design stable stabilizing controllers were first explored for MIMO plants that have at most two blocking-zeros on the extended non-negative real axis in [11], where connections to the sufficient conditions in [17] were also established. These results excluded plants that have transmission-zeros (instead of blocking-zeros) and plants that have more than a total of two zeros at the origin and infinity. In the special case of SISO plants, this implied that the results were not applicable for plants with relative degree larger than two. In this work, we obtain a stable stabilizing controller design procedure that applies to any strongly stabilizable plant with any number of zeros (transmission and blocking) at the origin and at infinity, and at most one finite positive real zero. Hence constraints of [11] on the number of zeros at the origin and at infinity are removed here and the results are generalized to include transmission-zeros as well as blocking-zeros. The plant class under consideration has no restrictions on the poles; zeros in the open left-half complex plane are also completely unrestricted. However, these plants have no unstable zeros except on the extended non-negative real axis.

Although other design methods are available for MIMO plants without restrictions on the unstable zeros, such methods assume other sufficient conditions in addition to PIP to obtain strongly stabilizing controllers (e.g., [1], [2], [4], [12]). For example, when the plant has two complex conjugate zeros located in such a way that the PIP is about to be violated (as the imaginary part goes to zero), many of the existing finite dimensional controller design techniques fail because in this case the minimum order of the strongly stabilizing controllers can be very large (grows as the imaginary part gets smaller) [13]. Our goal is to derive simple strongly stabilizing controllers without imposing additional conditions and hence, the design procedure developed here works for every plant satisfying the PIP in the class considered here. The proposed method also allows freedom in the design parameters, which may be used for additional performance objectives that are not considered here. It is shown using standard robustness arguments that the designed controllers provide robust closed-loop stability if the plant is subject to stable additive or pre-multiplicative perturbations. In the 
special case of SISO plants, the proposed design method leads to a stable stabilizing controller whose order is one less than the order of the given plant.

The paper is organized as follows: Section II gives the problem formulation, and defines the class of plants considered for strong stabilization. The main result in Section III, Theorem 1, provides a systematic procedure of constructing strongly stabilizing controllers for the class of MIMO plants considered. Concluding remarks are made in Section IV.

Although we discuss continuous-time systems here, all results apply also to discrete-time systems with appropriate modifications. The following fairly standard notation is used:

Notation: Let $\mathbb{R}, \mathbb{R}_{+}, \mathbb{C}$ denote real, positive real, and complex numbers, respectively. The extended closed righthalf plane is $\mathcal{U}=\{s \in \mathbb{C} \mid \mathcal{R} e(s) \geq 0\} \cup\{\infty\} ; \mathbf{R}_{\mathbf{p}}$ denotes real proper rational functions of $s ; \mathbf{S} \subset \mathbf{R}_{\mathbf{p}}$ is the stable subset with no poles in $\mathcal{U} ; \mathcal{M}(\mathbf{S})$ is the set of matrices with entries in $\mathbf{S} ; I$ is the identity matrix (of appropriate dimension). A transfer-matrix $M \in \mathcal{M}(\mathbf{S})$ is called unimodular iff $M^{-1} \in \mathcal{M}(\mathbf{S})$. The $H_{\infty}$-norm of $M \in \mathcal{M}(\mathbf{S})$ is denoted by $\|M\|$ (i.e., the norm $\|\cdot\|$ is the usual operator norm $\|M\|:=\sup _{s \in \partial \mathcal{U}} \bar{\sigma}(M(s))$, where $\bar{\sigma}$ is the maximum singular value and $\partial \mathcal{U}$ is the boundary of $\mathcal{U}$ ). For simplicity, we drop $(s)$ in transfer-matrices such as $G(s)$ where this causes no confusion. We use coprime factorizations over $\mathbf{S}$; i.e., for $P \in \mathbf{R}_{\mathbf{p}}{ }^{m \times m}, P=D^{-1} N$ denotes a left-coprimefactorization (LCF), where $N \in \mathbf{S}^{m \times m}, D \in \mathbf{S}^{m \times m}$, $\operatorname{det} D(\infty) \neq 0$. For full-rank $P$, we say that $z \in \mathcal{U}$ is a $\mathcal{U}$-zero of $P$ if $\operatorname{rank} N(z)<m$; these zeros include both transmission-zeros and blocking-zeros in $\mathcal{U}$. In the product notation frequently used throughout, it is assumed that

$$
\prod_{j=\nu}^{\eta} g_{j}=1 \quad \text { if } \quad \nu>\eta \text {. }
$$

\section{Problem Description and Plant Classes}

Consider the standard LTI, MIMO unity-feedback system $\operatorname{Sys}(P, C)$ shown in Fig. 1, where $P \in \mathbf{R}_{\mathbf{p}}{ }^{m \times m}$ and $C \in \mathbf{R}_{\mathbf{p}}{ }^{m \times m}$ denote the plant's and the controller's transfermatrices, respectively. It is assumed that the feedback system is well-posed, $P$ and $C$ have no unstable hidden-modes, and the plant $P \in \mathbf{R}_{\mathbf{p}}{ }^{m \times m}$ is full normal rank $m$. The objective is to design a stable stabilizing controller $C$.

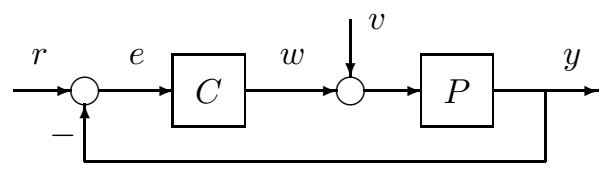

Fig. 1. Unity-Feedback System $\operatorname{Sys}(P, C)$.

Let $P=D^{-1} N$ be a left-coprime-factorization (LCF) of the plant and $C=N_{c} D_{c}^{-1}$ be a right-coprime-factorization (RCF) of the controller, where $N, D, N_{c}, D_{c} \in \mathcal{M}(\mathbf{S})$ have appropriate sizes, $\operatorname{det} D(\infty) \neq 0$, $\operatorname{det} D_{c}(\infty) \neq 0$. The system $\operatorname{Sys}(P, C)$ is said to be stable iff the closedloop transfer-function from $(r, v)$ to $(y, w)$ is stable. The controller $C$ is said to stabilize $P$ iff $C$ is proper and the system $\operatorname{Sys}(P, C)$ is stable. The controller $C$ stabilizes $P \in \mathcal{M}\left(\mathbf{R}_{\mathbf{p}}\right)$ if and only if

$$
M:=D D_{c}+N N_{c}
$$

is unimodular [14], [10]. Moreover, the stabilizing controller $C$ is stable if and only if $M$ in (1) is unimodular with a unimodular $D_{c}$; in this case $C$ is said to strongly stabilize $P$. There exist strongly stabilizing controllers for a given plant $P$ if and only if $P$ satisfies the PIP. Let $z_{1}, \ldots, z_{\ell} \in$ $\mathbb{R} \cap \mathcal{U}$ be the non-negative real-axis blocking-zeros of $P$ in the extended closed right-half-plane, i.e., $N\left(z_{k}\right)=0$ for $1 \leq k \leq \ell$. Then $P$ satisfies the PIP if and only if $\operatorname{det} D\left(z_{k}\right)$ is sign invariant for $1 \leq k \leq \ell$ (see e.g., [14]).

The plants under consideration here for strongly stabilizing controller synthesis have no restrictions on their poles; there are no restrictions on the zeros in the open left-half complex plane $\mathbb{C} \backslash \mathcal{U}$, at the origin $s=0$, and at infinity. However, the finite non-zero $\mathcal{U}$-zeros are restricted. We only consider the case where the plant $P$ has at most one non-zero finite zero in the region of instability $\mathcal{U}$ and it does not have a pole at that same point in $\mathcal{U}$. At the $\mathcal{U}$-zeros of $P$, the numerator $N$ in any LCF $P=D^{-1} N$ drops rank; i.e., $z \in \mathcal{U}$ is a $\mathcal{U}$-zero if $\operatorname{rank} N(z)<m$. Write $N^{-1}$ as

$$
N^{-1}=\left[\frac{x_{i j}}{y_{i j}}\right]_{i, j=1, \ldots m}
$$

where $x_{i j}, y_{i j} \in \mathbf{S}, i, j=1, \ldots m$. Then the largest numerator invariant-factor $\lambda_{z} \in \mathbf{S}$ is a least-common-multiple of all $y_{i j}$, and hence, $\lambda_{z} N^{-1} \in \mathcal{M}(\mathbf{S})$. If $P$ has a non-zero $\mathcal{U}$-zero, then the general expression for the largest invariantfactor $\lambda_{z}$ of $N$ is

$$
\lambda_{z}=\frac{(1-s / z)}{(s+a)}\left(\prod_{i=1}^{n_{\infty}} \frac{1}{\left(s+a_{i}\right)}\right)\left(s^{n_{o}} \prod_{j=1}^{n_{o}} \frac{1}{\left(s+b_{j}\right)}\right),
$$

where $a \in \mathbb{R}_{+}, a_{i} \in \mathbb{R}_{+}$for $1 \leq i \leq n_{\infty}, b_{j} \in \mathbb{R}_{+}$ for $1 \leq j \leq n_{o}$. The total number of $\mathcal{U}$-zeros of $\lambda_{z}$ is $n=n_{\infty}+n_{o}+1$, where $n_{o}$ is the number of zeros at the origin $s=0$, and $n_{\infty}$ is the number of zeros at infinity; if the plant has no finite positive real zeros but has zeros at infinity, then in (3), $z=\infty$ as well and the number of zeros of $\lambda_{z}$ at $s=\infty$ is $n_{\infty}+1$. If the plant has no zeros at infinity and has one finite positive zero, then the expression (3) is still valid with $n_{\infty}=0$. If the $\mathcal{U}$-zeros of $P$ are only at the origin, and it has no finite positive zeros and no zeros at infinity, then the term $\frac{(1-s / z)}{(s+a)}$ in (3) is replaced with $\frac{s}{(s+a)}$ and hence, the expression for $\lambda_{z}$ in (3) becomes

$$
\lambda_{z}=\frac{s}{(s+a)} s^{n_{o}} \prod_{j=1}^{n_{o}} \frac{1}{\left(s+b_{j}\right)},
$$

where the number of zeros of $\lambda_{z}$ at $s=0$ is $n_{o}+1=n$. Some examples of plants in this class are as follows: The 
plant $P_{1}$ has $\mathcal{U}$-zeros at $z=2$ and at $s=\infty$, with $n_{\infty}=1$ and $n_{o}=0$, i.e., $n=2$ :

$$
P_{1}=\left[\begin{array}{cc}
\frac{2 s^{2}+26 s+100}{(s-5)(s+6)} & \frac{-s^{3}+s^{2}+83 s+12}{(s+3)(s-5)(s+6)} \\
\frac{(s-2)(s+3)}{(s+4)\left(s^{2}+9\right)} & \frac{s-2}{s^{2}+9}
\end{array}\right] .
$$

The plant $P_{2}$ has $\mathcal{U}$-zeros at $s=0$ and at $s=\infty$; since it has no finite non-zero zero, we would consider $z=\infty$ and hence, $n_{\infty}=0, n_{o}=1$, i.e., $n=2$ :

$$
P_{2}=\left[\begin{array}{ll}
\frac{(s+2)(s-3)}{(s+5)(s-4)} & \frac{(s+2)(4 s-1)}{(s+5)(s-4)} \\
\frac{3(s+2)}{(s+1)(s-3)} & \frac{s+2}{(s+1)(s-3)}
\end{array}\right] \text {. }
$$

Let $P_{3}=\left[\begin{array}{cc}P_{1} & G \\ 0 & P_{2}\end{array}\right]$, where $G$ can be any stable $2 \times 2$ matrix; $P_{3}$ has $\mathcal{U}$-zeros at $z=2$, at $s=\infty$ and at $s=0$, with $n_{\infty}=1, n_{o}=1$, i.e., $n=3$. Let $P_{4}=\frac{s}{y(s)} P_{1}$, where $y(s)$ is any polynomial and $r$ is the relative degree of $\frac{s}{y(s)} ; P_{4}$ has $\mathcal{U}$-zeros at $z=2$, at $s=\infty$ and at $s=0$, with $n_{\infty}=r+1$, $n_{o}=1, n=r+3$. On the other hand, $P_{5}=\frac{s}{y(s)} P_{2}$ has $\mathcal{U}$-zeros at $s=\infty$ and at $s=0$, with $n_{\infty}=r, n_{o}=2$, $n=r+3$. The plants $P_{4}$ and $P_{5}$ have blocking-zeros at $s=\infty$ and $s=0$, whereas all $\mathcal{U}$-zeros in $P_{1}, P_{2}, P_{3}$ are transmission-zeros.

In Section III we propose a set of strongly stabilizing controllers for the plant class described with $\lambda_{z}$ as in (3) when the $\mathcal{U}$-zeros are at $s=z, 0, \infty$, or as in (4) when the $\mathcal{U}$-zeros are all at $s=0$.

\section{Strongly Stabilizing Controllers}

Theorem 1 gives a systematic strongly stabilizing controller design method for the plant class described in Section II. It is assumed that the plants under consideration are strongly stabilizable, and have at most one $\mathcal{U}$-zero $z \in \mathcal{U}$ but they do not have a coinciding pole at the same $z \in \mathcal{U}$. Therefore, $D(z)$ is non-singular for any LCF $P=D^{-1} N$.

Theorem 1: (Strongly stabilizing controller synthesis) Let $P \in \mathbf{R}_{\mathbf{p}}{ }^{m \times m}$ be strongly stabilizable and be described with $\lambda_{z}$ as in (3) or (4), where $D(z)$ is non-singular. If $n_{\infty} \neq 0$, then assume that all eigenvalues of $W:=D(z)^{-1} D(\infty)$ have positive real parts. For $\ell=2, \ldots, n_{\infty}$, define

$$
\Gamma_{\ell}:=\prod_{i=\ell}^{n_{\infty}} \frac{\alpha_{i}}{s+\alpha_{i}}
$$

if $\ell>n_{\infty}$, then $\Gamma_{\ell}=1$. Choose $\alpha_{i} \in \mathbb{R}_{+}$such that

$$
\alpha_{1}>\left\|s\left(D D(\infty)^{-1}-I\right)\right\|,
$$

and for $i=2, \ldots, n_{\infty}$, choose $\alpha_{i} \in \mathbb{R}_{+}$such that

$$
\alpha_{i}>\left\|s\left(D D(z)^{-1}-I\right) \Phi_{i}\right\|,
$$

where $\Phi_{i} \in \mathcal{M}(\mathbf{S})$ is defined as

$\Phi_{i}:=$

$$
\left[I-D D(z)^{-1}+D\left(s I+\alpha_{1} W\right) D(\infty)^{-1} \frac{1}{\alpha_{1}} \prod_{\nu=2}^{i-1} \frac{s+\alpha_{\nu}}{\alpha_{\nu}}\right]^{-1}
$$

If $n_{\infty}=0$, let $U_{n_{\infty}}:=D(z)$; if $n_{\infty} \neq 0$, let $U_{n_{\infty}}$ be

$$
U_{n_{\infty}}:=D+(D(\infty)-D W)\left(s I+\alpha_{1} W\right)^{-1} \alpha_{1} \Gamma_{2} .
$$

If $n_{o} \neq 0$, then assume that all eigenvalues of $\hat{W}:=$ $D(0)^{-1} D(z)$ have positive real parts. For $\ell=2, \ldots, n_{o}$ define

$$
\hat{\Gamma}_{\ell}:=\prod_{j=\ell}^{n_{o}} \frac{1}{s+\beta_{j}}
$$

if $\ell>n_{o}$, then $\hat{\Gamma}_{\ell}=1$. Choose $\beta_{1} \in \mathbb{R}_{+}$such that

$$
\beta_{1}<\left\|s^{-1}\left(D \hat{W} U_{n_{\infty}}^{-1}-I\right)\right\|^{-1}
$$

and for $j=2, \ldots, n_{o}$, choose $\beta_{j} \in \mathbb{R}_{+}$such that

$$
\beta_{j}<\left\|s^{-1}\left(D D(z)^{-1}-I\right) \Psi_{j}\right\|^{-1},
$$

where $\Psi_{j} \in \mathcal{M}(\mathbf{S})$ is defined as

$$
\Psi_{j}:=\left[I-D D(z)^{-1}+D \frac{\left(s I+\beta_{1} \hat{W}\right)}{s^{j-1}} E_{n_{\infty}} \prod_{\nu=2}^{j-1}\left(s+\beta_{\nu}\right)\right]^{-1},
$$

and

$$
E_{n_{\infty}}= \begin{cases}D(z)^{-1} & \text { if } n_{\infty}=0 \\ \frac{1}{\alpha_{1}}\left(s I+\alpha_{1} W\right) D(\infty)^{-1} \Gamma_{2}^{-1} & \text { if } n_{\infty} \neq 0\end{cases}
$$

Then the stable controller

$$
C=N^{-1}(D(z)-D) F_{\infty} F_{o}
$$

strongly stabilizes $P$, where $F_{\infty}$ and $F_{o}$ are given by

$$
\begin{aligned}
& F_{\infty}= \begin{cases}I & \text { if } n_{\infty}=0 \\
\alpha_{1} W\left(s I+\alpha_{1} W\right)^{-1} \Gamma_{2} & \text { if } n_{\infty} \neq 0\end{cases} \\
& F_{o}= \begin{cases}I & \text { if } n_{o}=0 \\
s^{n_{o}}\left(s I+\beta_{1} \hat{W}\right)^{-1} \hat{\Gamma}_{2} & \text { if } n_{o} \neq 0\end{cases}
\end{aligned}
$$

Furthermore, with $C \in \mathcal{M}(\mathbf{S})$ as in (15), the controller

$$
C_{q}=C+Q
$$

also strongly stabilizes $P$ for all $Q \in \mathbf{S}^{m \times m}$ such that

$$
\|Q\|<\left\|(I+P C)^{-1} P\right\|^{-1} \text {. }
$$

Remark (The order of the proposed controllers): In the case of SISO plants, the order of the controller $C$ in (15) is one less than the plant's order. Although coprime factorizations are unique only up to a unit in $\mathbf{S}$, without loss of generality it can be assumed that the chosen numerator in the factorization $P=D^{-1} N$ is in the form of the largest invariant-factor $\lambda_{z}$ given in (3) or (4). For purposes of discussing the order, we write the numerator and denominator factors of the plant in polynomial form as:

$$
P=\frac{(1-s / z) s^{n_{o}} \eta}{d},
$$

where $\eta$ is an $\tilde{n}$-th order polynomial whose roots are the zeros of the plant in the stable region $\mathbb{C} \backslash \mathcal{U}$, and $d$ is a 
polynomial of degree $\delta=n_{\infty}+n_{o}+\tilde{n}+1$. Then a coprime factorization $P=D^{-1} N$ over $\mathbf{S}$ is given by

$$
\begin{gathered}
D=\frac{d}{(1-s / z) s^{n_{o}} \eta} \lambda_{z} \\
=\frac{d}{\eta(s+a) \prod_{i=1}^{n_{\infty}}\left(s+a_{i}\right) \prod_{j=1}^{n_{o}}\left(s+b_{j}\right)}, \\
N=\lambda_{z}=\frac{(1-s / z) s^{n_{o}}}{(s+a) \prod_{i=1}^{n_{\infty}}\left(s+a_{i}\right) \prod_{j=1}^{n_{o}}\left(s+b_{j}\right)} .
\end{gathered}
$$

Using $D, N$ given by (21)-(22), the controller $C$ in (15) becomes

$$
\begin{gathered}
C=\lambda_{z}^{-1}(D(z)-D) F_{\infty} F_{o} \\
=\frac{\left[D(z)(s+a) \prod_{i=1}^{n_{\infty}}\left(s+a_{i}\right) \prod_{j=1}^{n_{o}}\left(s+b_{j}\right)-d\right] \alpha_{1} W s^{n_{o}}}{(1-s / z) s^{n_{o}} \eta\left(s+\alpha_{1} W\right)\left(s+\beta_{1} \hat{W}\right) \Gamma_{2}^{-1} \hat{\Gamma}_{2}^{-1}},
\end{gathered}
$$

where the numerator of $(D(z)-D)$ has a zero at $s=z$ and hence, cancels the term $(1-s / z)$ from the denominator of $C$. The polynomial terms $\eta\left(s+\alpha_{1} W\right)\left(s+\beta_{1} \hat{W}\right) \Gamma_{2}^{-1} \hat{\Gamma}_{2}^{-1}$ that remain in the denominator after cancelations have order $\tilde{n}+n_{\infty}+n_{o}$, where the degree of $\Gamma_{2}^{-1}$ is $n_{\infty}-1$ and the degree of $\hat{\Gamma}_{2}^{-1}=n_{o}-1$. Therefore, the order of the controller $C$ is $\tilde{n}+n_{\infty}+n_{o}=\delta-1$, where $\delta$ is the order of the plant.

We showed that the controller order is one less than the plant order for the case where the plant has at least one non-zero zero on the extended non-negative real-axis so that $\lambda_{z}$ is as in (3). Using entirely similar steps, it can be concluded that the controller order is again one less than the plant order when $\lambda_{z}$ is given by (4).

Remark (Robustness of the proposed strongly stabilizing controllers): Under the assumptions of Theorem 1, let the stable controller $C$ be given by (15) and let $C_{q}=C+Q$ for any $Q \in \mathbf{S}^{m \times m}$ satisfying (19). Then standard robustness arguments lead to the following conclusions (e.g., [14], [18]): a) (Additive perturbations): Let $\Delta \in \mathbf{S}^{m \times m}$ be such that

$$
\|\Delta\|<\left\|\left(I+C_{q} P\right)^{-1} C_{q}\right\|^{-1} .
$$

Then the controller $C_{q}$ strongly stabilizes $P+\Delta$ for all $\Delta \in$ $\mathbf{S}^{m \times m}$ satisfying (23).

b) (Multiplicative perturbations): Let $\Delta \in \mathbf{S}^{m \times m}$ be such that

$$
\|\Delta\|<\left\|\left(I+C_{q} P\right)^{-1} C_{q} P\right\|^{-1} .
$$

Then the controller $C_{q}$ strongly stabilizes $P(I+\Delta)$ for all $\Delta \in \mathbf{S}^{m \times m}$ satisfying (24).

c) (Coprime factor perturbations): Let $\Delta_{N}, \Delta_{D} \in \mathbf{S}^{m \times m}$ be such that

$$
\left\|\left[\begin{array}{ll}
\Delta_{D} & \Delta_{N}
\end{array}\right]\right\|<\left\|\left[\begin{array}{c}
C_{q} \\
I
\end{array}\right] M^{-1}\right\|^{-1} .
$$

where $M=D+N C_{q}$ is unimodular by design for all $C_{q}=$ $C+Q$ with $Q$ satisfying (19). Then the controller $C_{q}$ strongly stabilizes all plants in the form $\left(D+\Delta_{D}\right)^{-1}\left(N+\Delta_{N}\right)$ satisfying (25).

Once $C$ is fixed, one can try to optimize $Q$ to maximize the allowable perturbation magnitude (23) or (25). This problem can be formulated as a two block $H_{\infty}$ control problem as follows: Consider the robustness optimization for coprime factor perturbation (25). First note that when there is no uncertainty and $C_{q}=C+Q$ is used as the controller, the feedback system is stable if and only if $(D+N C+N Q)^{-1} \in$ $\mathbf{S}^{m \times m}$. Since by design $M=D+N C$ is unimodular, the feedback system with plant $P=D^{-1} N$ and controller $C_{q}=C+Q$ is stable if

$$
\left\|M^{-1} N Q\right\|<1 .
$$

Therefore, to maximize the left hand side of (25) we want to minimize

$$
\left\|\left[\begin{array}{c}
C+Q \\
I
\end{array}\right] M^{-1}\right\|
$$

over all $Q \in \mathbf{S}^{m \times m}$ satisfying (26). A slightly conservative way to solve this problem is to minimize $\gamma>0$ in

$$
\left\|\gamma^{-1}\left[\begin{array}{c}
C \\
0 \\
I
\end{array}\right] M^{-1}+\left[\begin{array}{c}
\gamma^{-1} I \\
N \\
0
\end{array}\right] Q M^{-1}\right\|<1
$$

over all $Q \in \mathbf{S}^{m \times m}$, which is a two-block $H_{\infty}$ control problem and can be solved using standard techniques, [18].

Similar arguments show that when we have additive uncertainty, to maximize the left hand side of (23) we want to minimize $\gamma>0$ subject to

$$
\left\|\gamma^{-1}\left[\begin{array}{l}
C \\
0
\end{array}\right] M^{-1}+\left[\begin{array}{c}
\gamma^{-1} I \\
N
\end{array}\right] Q M^{-1}\right\|<1
$$

over all $Q \in \mathbf{S}^{m \times m}$, which is a slightly simpler two block $H_{\infty}$ control problem.

\section{Conclusions}

We proposed a simple strongly stabilizing controller synthesis method for a class of unstable MIMO plants satisfying the PIP, with at most one positive real zero and any number of zeros at $s=0$, at infinity, and in the open left-half complex plane. No restrictions were imposed on the number and locations of the poles. We explicitly constructed robust strongly stabilizing controllers for all plants in this class. The design offers freedom in the design parameters that may be used for other performance criteria. In the special case of SISO plants, the order of the (nominal) strongly stabilizing controller obtained using the proposed design procedure here is one less than the order of the plant.

The more challenging problem of strongly stabilizing controller design for MIMO plants with more than one positive real zero and complex conjugate zero pairs is to be tackled in our future study using the method developed here. In the SISO plant case, the order of the controller is a few multiples of the plant order in many of the existing finite dimensional controller design methods and the order grows as the positive pole/zero locations are close to violating the PIP. Therefore, it is expected that the extension of the results presented here will involve much less simpler designs when we consider plants with two or more finite non-zero zeros. The results presented here are important in identifying plant classes that can be strongly stabilized using the proposed 
design method resulting in simple stable controllers without the added sufficient conditions assumed in alternate methods.

\section{APPENDIX}

Proof of Theorem 1: We first show that the controller proposed in (15) is stable. By assumption, the largest numerator invariant-factor $\lambda_{z} \in \mathbf{S}$ is as in either (3) or (4). Let $\lambda_{z} N^{-1}=: N_{s}$; then $N_{s} \in \mathcal{M}(\mathbf{S})$ and $N^{-1}=\lambda_{z}^{-1} N_{s}$. We have to show that

$$
\begin{aligned}
& C= N^{-1}(D-D(z)) F_{\infty} F_{o} \\
&=N_{s} \lambda_{z}^{-1}(D-D(z)) F_{\infty} F_{o} \\
&=N_{s} \lambda_{z}^{-1}(D-D(z)) \alpha_{1} W\left(s I+\alpha_{1} W\right)^{-1} \Gamma_{2} \\
& \cdot s^{n_{o}}\left(s I+\beta_{1} W\right)^{-1} \hat{\Gamma}_{2} \in \mathcal{M}(\mathbf{S}) .
\end{aligned}
$$

Since $(D(s)-D(z))$ is zero at $s=z$, the term $\frac{(s+a)}{(1-s / z)}(D(s)-D(z))$ is stable. If $n_{\infty} \neq 0$, then

$$
\left(\prod_{i=1}^{n_{\infty}}\left(s+a_{j}\right)\right)\left(\alpha_{1} W\left(s I+\alpha_{1} W\right)^{-1} \Gamma_{2}\right) \in \mathbf{S} .
$$

If $n_{o} \neq 0$, then

$$
\left(s^{-n_{o}} \prod_{j=1}^{n_{o}}\left(s+b_{j}\right)\right)\left(s^{n_{o}}\left(s I+\beta_{1} \hat{W}\right)^{-1} \hat{\Gamma}_{2}\right) \in \mathbf{S} .
$$

Note that if there is no finite positive zero, but $P$ has blocking-zeros at infinity, we take $z=\infty$; hence, $W=$ $I$. If all $\mathcal{U}$-zeros in $P$ are at $s=0$, then the term $\frac{(1-s / z)}{(s+a)}$ in $N_{z}$ is replaced with $\frac{s}{(s+a)}$ as in (4). In this case, $F_{\infty}=I, \hat{W}=I, \frac{s}{(s+a)}(D(s)-D(0)) \in \mathcal{M}(\mathbf{S})$, $\left(s^{-n_{o}} \prod_{j=1}^{n_{o}}\left(s+b_{j}\right)\right)\left(s^{n_{o}}\left(s I+\beta_{1} \hat{W}\right)^{-1} \hat{\Gamma}_{2}\right) \in \mathbf{S}$. Therefore, in all cases the proposed controller in (15) is stable. It remains to show that the proposed $C$ stabilizes $P$ :

Step 1: Let $N_{c}=C$ and $D_{c}=I$; by (1), $C=N_{c} D_{c}^{-1}$ stabilizes $P=D^{-1} N$ if and only if $M=D+N C$ is unimodular. With $U_{o}:=D(z)$, write $M=D+N N^{-1}(D(z)-$ $D) F_{\infty} F_{o}=U_{o} F_{\infty} F_{o}+D\left(I-F_{\infty} F_{o}\right)$. If $n_{\infty}=0$, then $F_{\infty}=I$; go to step 2. If $n_{\infty}>0$, write $M$ as

$$
\begin{gathered}
M=D+(D(z)-D) \alpha_{1} W\left(s I+\alpha_{1} W\right)^{-1} \Gamma_{2} F_{o} \\
=\left(\alpha_{1} D(z) W+s D\right)\left(s I+\alpha_{1} W\right)^{-1} \Gamma_{2} F_{o}+D\left(I-\Gamma_{2} F_{o}\right) \\
=U_{1} \Gamma_{2} F_{o}+D\left(I-\Gamma_{2} F_{o}\right),
\end{gathered}
$$

where $U_{1}$ is defined as

$$
\begin{aligned}
& U_{1}:=\left(\frac{\alpha_{1}}{s+\alpha_{1}} D(\infty)+\frac{s}{s+\alpha_{1}} D\right)\left(s+\alpha_{1}\right)\left(s I+\alpha_{1} W\right)^{-1} \\
& =\left[I+\frac{1}{s+\alpha_{1}} s\left(D D(\infty)^{-1}-I\right)\right] D(\infty)\left(s+\alpha_{1}\right)\left(s I+\alpha_{1} W\right)^{-1} .
\end{aligned}
$$

Then $\left(D D(\infty)^{-1}-I\right)$ strictly-proper implies $s\left(D D(\infty)^{-1}-\right.$ $I) \in \mathcal{M}(\mathbf{S})$, and hence, $U_{1}$ is unimodular for $\alpha_{1}$ satisfying
(6). If $n_{\infty}=1$, then $\Gamma_{2}=1$; go to step 2. If $n_{\infty}>1$, write $M$ as

$$
\begin{aligned}
M=U_{1} \frac{\alpha_{2}}{s+\alpha_{2}} \Gamma_{3} F_{o}+D & \left(I-\frac{\alpha_{2}}{s+\alpha_{2}} \Gamma_{3} F_{o}\right) \\
& =U_{2} \Gamma_{3} F_{o}+D\left(I-\Gamma_{3} F_{o}\right),
\end{aligned}
$$

where $U_{2}$ is defined as

$$
\begin{aligned}
& U_{2}:=\frac{\alpha_{2}}{s+\alpha_{2}} U_{1}+\frac{s}{s+\alpha_{2}} D \\
& \quad=\left[I+\frac{1}{s+\alpha_{2}} s\left(D U_{1}^{-1}-I\right)\right] U_{1} \\
& =D+(D(\infty)-D W)\left(s I+\alpha_{1} W\right)^{-1} \alpha_{1} \frac{\alpha_{2}}{s+\alpha_{2}} .
\end{aligned}
$$

For $i=2, \prod_{\nu=2}^{i-1} \frac{s+\alpha_{\nu}}{\alpha_{\nu}}=1$ in (8); then $\Phi_{2}=$ $\left(I-D D(z)^{-1}+D \frac{1}{\alpha_{1}}\left(s I+\alpha_{1} W\right) D(\infty)^{-1}\right)^{-1}=$ $\left(I+\frac{s}{\alpha_{1}} D D(\infty)^{-1}\right)^{-1}$ implies that (7) becomes $\alpha_{2}>$ $\left\|s\left(D D(z)^{-1}-I\right) \Phi_{2}\right\|=\| s\left(D D(z)^{-1}-I\right)(I+$ $\left.\frac{s}{\alpha_{1}} D D(\infty)^{-1}\right)^{-1}\|=\| s\left(D U_{1}^{-1}-I\right) \|$. Therefore, $U_{2}$ is unimodular for $\alpha_{2}$ satisfying (7). If $n_{\infty}=2$, then $\Gamma_{3}=1$; go to step 2. If $n_{\infty}>2$, write $M$ as

$$
\begin{aligned}
M=U_{2} \frac{\alpha_{3}}{s+\alpha_{3}} \Gamma_{4} F_{o}+D & \left(I-\frac{\alpha_{3}}{s+\alpha_{3}} \Gamma_{4} F_{o}\right) \\
& =U_{3} \Gamma_{4} F_{o}+D\left(I-\Gamma_{4} F_{o}\right),
\end{aligned}
$$

where $U_{3}$ is defined as

$$
\begin{aligned}
& U_{3}:=\frac{\alpha_{3}}{s+\alpha_{3}} U_{2}+\frac{s}{s+\alpha_{3}} D \\
& \quad=\left[I+\frac{1}{s+\alpha_{3}} s\left(D U_{2}^{-1}-I\right)\right] U_{2} \\
& =D+(D(\infty)-D W)\left(s I+\alpha_{1} W\right)^{-1} \alpha_{1} \prod_{i=2}^{3} \frac{\alpha_{i}}{s+\alpha_{i}} .
\end{aligned}
$$

For $i=3$, by (8), $\Phi_{3}=\left(I-D D(z)^{-1}+D \frac{1}{\alpha_{1}}(s I+\right.$ $\left.\left.\alpha_{1} W\right) D(\infty)^{-1} \frac{\left(s+\alpha_{2}\right)}{\alpha_{1} \alpha_{2}}\right)^{-1}$ implies (7) becomes $\alpha_{3}>$ $\left\|s\left(D D(z)^{-1}-I\right) \Phi_{3}\right\|=\left\|s\left(D U_{2}^{-1}-I\right)\right\|$. Therefore, $U_{3}$ is unimodular for $\alpha_{3}$ satisfying (7). If $n_{\infty}=3$, then $\Gamma_{4}=1$; go to step 2. If $n_{\infty}>3$, then continue similarly with $U_{k}$ defined as

$$
U_{k}=D+(D(\infty)-D W)\left(s I+\alpha_{1} W\right)^{-1} \alpha_{1} \prod_{i=2}^{k} \frac{\alpha_{i}}{s+\alpha_{i}} .
$$

Write $M$ as

$$
\begin{array}{r}
M=U_{k} \frac{\alpha_{k+1}}{s+\alpha_{k+1}} \Gamma_{k+2} F_{o}+D\left(I-\frac{\alpha_{k+1}}{s+\alpha_{k+1}} \Gamma_{k+2} F_{o}\right) \\
=U_{k+1} \Gamma_{k+2} F_{o}+D\left(I-\Gamma_{k+2} F_{o}\right),
\end{array}
$$

where $U_{k+1}$ is defined as

$$
\begin{aligned}
U_{k+1}:=\frac{\alpha_{k+1}}{s+\alpha_{k+1}} U_{k}+\frac{s}{s+\alpha_{k+1}} D \\
=\left[I+\frac{1}{s+\alpha_{k+1}} s\left(D U_{k}^{-1}-I\right)\right] U_{k} .
\end{aligned}
$$


s For $i=k+1$, by (8), $\Phi_{k+1}=\left(I-D D(z)^{-1}+\right.$ $\left.D \frac{1}{\alpha_{1}}\left(s I+\alpha_{1} W\right) D(\infty)^{-1} \prod_{\nu=2}^{k} \frac{\left(s+\alpha_{\nu}\right)}{\alpha_{\nu}}\right)^{-1}$ implies (7) becomes $\alpha_{k+1}>\left\|s\left(D D(z)^{-1}-I\right) \Phi_{k+1}\right\|=\left\|s\left(D U_{k}^{-1}-I\right)\right\|$. Therefore, $U_{k+1}$ is unimodular for $\alpha_{k+1}$ satisfying (7). If $n_{\infty}=k+1$, then $\Gamma_{k+2}=1$ and $M=U_{n_{\infty}} F_{o}+D\left(I-F_{o}\right)$, where $U_{n_{\infty}}$ is unimodular; go to step 2 .

Step 2: If $n_{o}=0$, then $F_{o}=I$; go to step 3. If $n_{o}>0$, write $M$ as

$$
\begin{array}{r}
M=U_{n_{\infty}} s\left(s I+\beta_{1} \hat{W}\right)^{-1} \hat{\Gamma}_{2}+D\left(I-s\left(s I+\beta_{1} \hat{W}\right)^{-1} \hat{\Gamma}_{2}\right) \\
=V_{1} \hat{\Gamma}_{2}+D\left(I-\hat{\Gamma}_{2}\right)
\end{array}
$$

where $U_{n_{\infty}}=D(z)$ if $n_{\infty}=0$ and $U_{n_{\infty}}$ is given by (9) if $n_{\infty} \neq 0$. Let $V_{1}$ be defined as

$$
\begin{aligned}
& V_{1}=\left(\frac{s}{s+\beta_{1}} U_{n_{\infty}}+\frac{\beta_{1}}{s+\beta_{1}} D \hat{W}\right)\left(s+\beta_{1}\right)\left(s I+\beta_{1} \hat{W}\right)^{-1} \\
= & {\left[I+\frac{\beta_{1} s}{s+\beta_{1}} s^{-1}\left(D \hat{W} U_{n_{\infty}}^{-1}-I\right)\right] U_{n_{\infty}}\left(s+\beta_{1}\right)\left(s I+\beta_{1} \hat{W}\right)^{-1} . }
\end{aligned}
$$

Then $\left[D \hat{W} U_{n_{\infty}}^{-1}-I\right]$ is zero at $s=0$ implies $s^{-1}\left(D \hat{W} U_{n_{\infty}}^{-1}-\right.$ $I) \in \mathcal{M}(\mathbf{S})$ and hence, $V_{1}$ is unimodular for $\beta_{1}$ satisfying (11). If $n_{o}=1$ then $\hat{\Gamma}_{2}=1$; go to step 3. If $n_{o}>1$, write $M$ as

$$
M=V_{1} \frac{s}{s+\beta_{2}} \hat{\Gamma}_{3}+D\left(1-\frac{s}{s+\beta_{2}} \hat{\Gamma}_{3}\right)=V_{2} \hat{\Gamma}_{3}+D\left(1-\hat{\Gamma}_{3}\right),
$$

where $V_{2}$ is defined as

$$
\begin{aligned}
& V_{2}= \frac{s}{s+\beta_{2}} V_{1}+\frac{\beta_{2}}{s+\beta_{2}} D \\
& \quad=\left[I+\frac{\beta_{2} s}{s+\beta_{2}}\left(s^{-1}\left[D V_{1}^{-1}-I\right]\right)\right] V_{1} \\
&=D+\left(U_{n_{\infty}}-D\right) s\left(s I+\beta_{1} \hat{W}\right)^{-1} \frac{s}{s+\beta_{2}} .
\end{aligned}
$$

For $j=2, \prod_{\nu=2}^{j-1}\left(s+\beta_{\nu}\right)=1$ in (13); then $\Psi_{2}=$ $\left(I-D D(z)^{-1}+1 s D\left(s I+\beta_{1} \hat{W}\right) E_{n_{\infty}}\right)^{-1}$ implies that (12) becomes $\beta_{2}<\left\|s^{-1}\left(D D(z)^{-1}-I\right) \Psi_{2}\right\|^{-1}=\| s^{-1}\left(D V_{1}^{-1}-\right.$ $I) \|^{-1}$. Therefore, $V_{2}$ is unimodular for $\beta_{2}$ satisfying (12). If $n_{o}=2$, then $\hat{\Gamma}_{3}=1$; go to step 3 . If $n_{o}>2$, then continue similarly with $V_{k}$ defined as

$$
V_{k}=D+\left(U_{n_{\infty}}-D\right) s\left(s I+\beta_{1} \hat{W}\right)^{-1} \prod_{j=2}^{k} \frac{s}{s+\beta_{j}} .
$$

Write $M$ as

$$
\begin{aligned}
M=V_{k} \frac{s}{s+\beta_{k+1}} \hat{\Gamma}_{k+2} & +D\left(I-\frac{s}{s+\beta_{k+1}} \hat{\Gamma}_{k+2}\right) \\
& =V_{k+1} \hat{\Gamma}_{k+2}+D\left(1-\hat{\Gamma}_{k+2}\right),
\end{aligned}
$$

where $V_{k+1}$ is defined as

$$
\begin{aligned}
V_{k+1}=\frac{s}{s+\beta_{k+1}} V_{k}+\frac{\beta_{k+1}}{s+\beta_{k+1}} D \\
=\left[I+\frac{\beta_{k+1} s}{s+\beta_{k+1}}\left(s^{-1}\left[D V_{k}^{-1}-I\right]\right)\right] V_{k} .
\end{aligned}
$$

For $j=k+1$, by (13), $\Psi_{k+1}=\left(I-D D(z)^{-1}+\right.$ $\left.D \frac{1}{s^{k}} E_{n_{\infty}} \prod_{\nu=2}^{k}\left(s+\beta_{\nu}\right)\right)^{-1}$ implies (12) becomes $\beta_{k+1}<$ $\left\|s^{-1}\left(D D(z)^{-1}-I\right) \Psi_{k+1}\right\|^{-1}=\left\|s^{-1}\left(D V_{k}^{-1}-I\right)\right\|^{-1}$. Therefore, $V_{k+1}$ is unimodular for $\beta_{k+1}$ satisfying (12). If $n_{o}=k+1$, then $\hat{\Gamma}_{k+2}=1$ and $M=V_{n_{o}}$ is unimodular; go to step 3 .

Step 3: If $n_{o}=0$, then $M=U_{n_{\infty}}$ is unimodular, where $U_{n_{\infty}}=U_{o}=D(z)$ if $n_{\infty}=0$ and $U_{n_{\infty}}$ is as in (9) if $n_{\infty} \neq 0$. If $n_{o}>0$, then $M=V_{n_{o}}$ is also unimodular. Since $M=D+N C$ is unimodular, the controller $C$ in (15) stabilizes $P=D^{-1} N$. The stable controller $C_{q}=C+Q$ also stabilizing the plant for $Q \in \mathcal{M}(\mathbf{S})$ satisfying (19) is standard 'small-gain' argument since $\left\|M^{-1} N Q\right\|=\left\|(D+N C)^{-1} N Q\right\|=\left\|(I+P C)^{-1} P Q\right\|<1$ implies $I+M^{-1} N Q$ is unimodular. Therefore, $M_{q}:=D+N C_{q}=(D+N C)+N Q=M+N Q=$ $M\left(I+M^{-1} N Q\right)$ is also unimodular, and hence, $C_{q} \in \mathcal{M}(\mathbf{S})$ also stabilizes $P$.

\section{REFERENCES}

[1] A. E. Barabanov, "Design of $H_{\infty}$ optimal stable controller," Proc. IEEE Conference on Decision and Control, pp. 734-738, 1996.

[2] D. U. Campos-Delgado, K. Zhou, "A parametric optimization approach to $\mathcal{H}^{\infty}$ and $\mathcal{H}^{2}$ strong stabilization," Automatica, vol. 39, no. 7, pp. 1205-1211, 2003.

[3] P. Cheng, Y.-Y. Cao, and Y. Sun, "On strong $\gamma_{k}-\gamma_{c l} H_{\infty}$ stabilization and simultaneous $\gamma_{k}-\gamma_{c l} H_{\infty}$ control," Proc. 46th IEEE Conference on Decision and Control, pp. 5417-5422, 2007.

[4] Y. S. Chou, J. L. Leu, and Y. C. Chu, "Stable controller design for MIMO systems: An LMI approach,” IET Control Theory Appl., Vol. 1, pp. 817-829, 2007.

[5] P. Dorato, H. Park, and Y. Li, "An algorithm with interpolation in units in $H^{\infty}$, with applications to feedback stabilization," Automatica, 25, pp. 427-430, 1989.

[6] N. H. El-Farra, P. Mhaskar, P. D. Christofides, "Hybrid predictive control of nonlinear systems: method and applications to chemical processes," Int. J. Robust Nonlinear Control, 14, 199-225, 2004.

[7] G. C. Goodwin, S. F. Graebe, M. E. Salgado, Control System Design, Prentice Hall, 2001.

[8] S. Gümüşsoy, H. Özbay, "Remarks on strong stabilization and stable $\mathcal{H}^{\infty}$ controller design," IEEE Transactions on Automatic Control, vol. 50, pp. 2083-2087, 2005.

[9] Y. Halevi, "Stable LQG controllers," IEEE Transactions on Automatic Control, vol. 39, pp. 2104-2106, 1994.

[10] A. N. Gündeş, C. A. Desoer, Algebraic Theory of Linear Feedback Systems with Full and Decentralized Compensators, Lect. Notes in Contr. and Inform. Sciences, 142, Springer, 1990.

[11] H. Özbay, A. N. Gündeş, "Strongly stabilizing controller synthesis for a class of MIMO plants," 17th IFAC World Congress, pp. 359-363, Seoul, Korea, 2008.

[12] I. Petersen, "Robust $\mathcal{H}^{\infty}$ control of an uncertain system via a strict bounded real output feedback controller," Proc. 45th IEEE Conference on Decision and Control, pp. 571-577, 2006.

[13] M. C. Smith, K. P. Sondergeld, "On the order of stable compensators," Automatica, vol. 22, pp. 127-129, 1986.

[14] M. Vidyasagar, Control System Synthesis: A Factorization Approach, Cambridge, MA: MIT Press, 1985.

[15] D. C. Youla, J. J. Bongiorno, and C. N. Lu, "Single-loop feedback stabilization of linear multivariable dynamical plants," Automatica, 10, pp. 159-173, 1974.

[16] M. Zeren, H. Özbay, "On the synthesis of stable $H_{\infty}$ controllers," IEEE Transactions on Automatic Control, vol. 44, pp. 431-435, 1999.

[17] M. Zeren, H. Özbay, "On the strong stabilization and stable $H_{\infty}$ controller design problems for MIMO systems," Automatica, vol. 36, pp. 1675-1684, 2000.

[18] K. Zhou, J. C. Doyle, and K. Glover, Robust and Optimal Control, Prentice-Hall, Upper Saddle River, NJ, 1996. 